\title{
THE RIESZ-DUNFORD AND WEYL FUNCTIONAL CALCULI
}

\author{
B. JEFFERIES
}

\begin{abstract}
Let $A_{1}, A_{2}$ be two bounded linear operators such that the spectrum $\sigma\left(A_{1} \xi+\right.$ $A_{2} \xi_{2}$ ) of every real linear combination $A_{1} \xi+A_{2} \xi_{2}$ of $A_{1}, A_{2}$ is real. We prove that the spectrum $\sigma\left(A_{1}+i A_{2}\right)$ of $A_{1}+i A_{2}$, considered as a subset of the plane $\mathbb{R}^{2}$, is contained in monogenic spectrum $\gamma\left(A_{1}, A_{2}\right)$ of the pair $\left(A_{1}, A_{2}\right)$. As a consequence, if $A_{1}, A_{2}$ are two bounded selfadjoint operators acting on a Hilbert space, then $\sigma\left(A_{1}+i A_{2}\right)$ is included in the support of the Weyl functional calculus for the pair $\left(A_{1}, A_{2}\right)$.
\end{abstract}

\section{INTRODUCTION}

In the Riesz-Dunford functional calculus, a function $f(T)$ of a single operator $T$ is defined by the Cauchy integral formula

$$
f(T)=\frac{1}{2 \pi i} \int_{C}(\lambda I-T)^{-1} f(\lambda) d \lambda,
$$

for a function $f$ holomorphic in a neighbourhood of the spectrum $\sigma(T)$ of $T$ and a simple closed contour $C$ about $\sigma(T)$. For two bounded selfadjoint operators on a Hilbert space $H$ or hermitian matrices $A=\left(A_{1}, A_{2}\right)$, the operator valued distribution

$$
\mathcal{W}_{A}: f \longmapsto \frac{1}{(2 \pi)^{2}} \int_{\mathbb{R}^{2}} e^{i\langle\xi, A\rangle} \hat{f}(\xi) d \xi, \quad f \in \mathcal{S}\left(\mathbb{R}^{2}\right),
$$

is the Weyl functional calculus for the pair $A=\left(A_{1}, A_{2}\right)$. In formula (2), for each $\zeta \in \mathbb{C}^{n}$, the operator $\sum_{j=1}^{n} \zeta_{j} A_{j}$ is denoted here by $\langle\zeta, A\rangle$, the Schwartz space of rapidly decreasing functions is denoted by $\mathcal{S}\left(\mathbb{R}^{2}\right)$ and $\hat{f}$ is the Fourier transform $\xi \longmapsto \int_{\mathbb{R}^{2}} e^{-i\langle x, \xi\rangle} f(x) d x, \xi \in \mathbb{R}^{2}$, of $f \in \mathcal{S}\left(\mathbb{R}^{2}\right)$. The operator version

Received 29 August 2006, revised 16 October 2006, accepted 18 October 2006.

2000 Mathematics Subject Classification: Primary 47A60, 46H30; Secondary 47A25, 30 G35

Key words and Phrases: spectrum, Weyl functional calculus, Clifford algebra, monogenic function 
of the Paley-Wiener theorem shows that the distribution $\mathcal{W}_{A}$ has support $\gamma(A)$ contained in the closed unit ball of radius $r=\sqrt{\left\|A_{1}\right\|^{2}+\left\|A_{2}\right\|^{2}}$ centred at zero in $\mathbb{R}^{2}$. Standard arguments of distribution theory enable a unique extension of the map defined by formula (2) to a distribution $\mathcal{W}_{A}: C^{\infty}\left(\mathbb{R}^{2}\right) \rightarrow \mathcal{L}(H)[1,9]$. The compact set $\gamma(A) \subset \mathbb{R}^{2}$ may be viewed as a type of joint spectrum for the possibly noncommuting operators $A_{1}, A_{2}$. If $A_{1}, A_{2}$ do commute, then $\mathcal{W}_{A}$ is actually the joint spectral measure of $A=\left(A_{1}, A_{2}\right)$ viewed as an operator valued distribution and $\gamma(A)$ is the joint spectrum of $A$.

Let $j$ denote the usual identification of $\mathbb{R}^{2}$ with $\mathbb{C}$ defined by $j\left(x_{1}, x_{2}\right)=x_{1}+$ $i x_{2}$ for $x_{1}, x_{2} \in \mathbb{R}^{2}$. It is natural to ask if the inclusion $\sigma\left(A_{1}+i A_{2}\right) \subset j \gamma(A)$ holds. Also, if $f$ is a holomorphic function defined in a neighbourhood of $j \gamma(A)$, does the linear operator $f\left(A_{1}+i A_{2}\right)$ defined by formula (1) equal $\mathcal{W}_{A}(f \circ j)$ ? It was shown in [1, Theorem 5.1] by an appeal to Runge's Theorem, that the answer is affirmative if $f$ is a holomorphic function defined in a simply connected neighbourhood of the union $\sigma\left(A_{1}+i A_{2}\right) \cup j \gamma(A)$. It was also proved in [1, Corollary 5.3] that the convex hull of $j \gamma(A)$ contains $\sigma\left(A_{1}+i A_{2}\right)$. In this note, the inclusion $\sigma\left(A_{1}+i A_{2}\right) \subset j \gamma(A)$ is established. It seems that the easiest route to this observation is by employing Clifford algebras, as follows.

Suppose that $\lambda \in \mathbb{C}$ and $\varphi_{\lambda}\left(x_{1}, x_{2}\right)=\left(\lambda-\left(x_{1}+i x_{2}\right)\right)^{-1}$ for all $x_{1}, x_{2} \in \mathbb{R}$ such that $x_{1}+i x_{2} \neq \lambda$. The inclusion $\sigma\left(A_{1}+i A_{2}\right) \subset j \gamma(A)$ is obviously a consequence of the formula

$$
\mathcal{W}_{A}\left(\varphi_{\lambda}\right)=\left(\lambda I-\left(A_{1}+i A_{2}\right)\right)^{-1}, \quad \lambda \in \mathbb{C} \backslash j \gamma(A)
$$

where we suppose here that the right-hand side makes sense of all $\lambda \in \mathbb{C} \backslash j \gamma(A)$. The left-hand side of equation (3) is defined because for each $\lambda \in \mathbb{C} \backslash j \gamma(A)$, the function $\varphi_{\lambda}$ is smooth in a neighbourhood of the support $\gamma(A)$ of the operator valued distribution $\mathcal{W}_{A}$. The equality $(3)$ is asserted in [3, Lemma 1], but there is a gap in the argument-precisely because the subset $\gamma(A)$ of the plane may have gaps or lacunas. The difficulty is that even if the equalities

$$
\left(\lambda I-\left(A_{1}+i A_{2}\right)\right) \mathcal{W}_{A}\left(\varphi_{\lambda}\right)=\mathcal{W}_{A}\left(\varphi_{\lambda}\right)\left(\lambda I-\left(A_{1}+i A_{2}\right)\right)=I
$$

are valid for $\lambda \in \mathbb{C}$ with $|\lambda|$ sufficiently large, there is no guarantee that the equalities hold for all $\lambda \in \mathbb{C} \backslash j \gamma(A)$ by analytic continuation, because $\mathbb{C} \backslash j \gamma(A)$ may have a bounded component as well as an unbounded component. An example of two $(3 \times 3)$ matrices in which this phenomenon occurs is exhibited in [7, Figure 3]. The remedy, given in the proof of Theorem 1 below, is to prove equation (4) for all large enough elements $\lambda$ of a higher dimensional complex algebra $\mathcal{A}$ in which $\mathbb{C}$ is embedded. Because the compact set $j \gamma(A) \subset \mathbb{C}$ cannot disconnect $\mathcal{A}$, formula (3) follows by analytic continuation. Although formula (3) makes no reference to Clifford algebras, a natural candidate for $\mathcal{A}$ is the four dimensional Clifford algebra $\mathbb{C}_{(2)}$ of complex quaternions.

In Theorem 1, a more general result is proved for bounded linear operators $A_{1}, A_{2}$ with the property that $\sigma\left(A_{1} \xi_{1}+A_{1} \xi_{2}\right)$ is real for all $\xi_{1}, \xi_{2} \in \mathbb{R}$, for example, 
selfadjoint operators on a Hilbert space. There is a functional calculus for such operators [4] using the techniques of Clifford analysis, to which we now turn.

\section{CLIFFORD ANALYSIS}

The great advantage of Clifford analysis over the theory of functions of several complex variables is apparent by comparing higher dimensional analogues of the Cauchy integral formula in each theory. For integral representation formulae of functions of several complex variables, see [8]. Let $\mathbb{C}_{(n)}$ be the Clifford algebra generated over the field $\mathbb{C}$ by the standard basis vectors $e_{0}, e_{1}, \ldots, e_{n}$ of $\mathbb{R}^{n+1}$ with conjugation $u \longmapsto \bar{u}$. When $n=2$, we obtain the 4-dimensional complex algebra of quaternions spanned by $\left(e_{0}, e_{1}, e_{2}, e_{1} e_{2}\right)=(1, i, j, k)$.

The generalized Cauchy-Riemann operator is given by $D=\sum_{j=0}^{n} e_{j} \frac{\partial}{\partial x_{j}}$. Let $U \subset \mathbb{R}^{n+1}$ be an open set. A function $f: U \rightarrow \mathbb{C}_{(n)}$ is called left monogenic if $D f=0$ in $U$ and right monogenic if $f D=0$ in $U$. The Cauchy kernel is given by

$$
G_{\omega}(x)=\frac{1}{\sigma_{n}} \frac{\overline{\omega-x}}{|\omega-x|^{n+1}}, \quad \omega, x \in \mathbb{R}^{n+1}, \omega \neq x,
$$

with $\sigma_{n}=2 \pi^{\frac{n+1}{2}} / \Gamma\left(\frac{n+1}{2}\right)$ the volume of unit $n$-sphere in $\mathbb{R}^{n+1}$. So, given a left monogenic function $f: U \rightarrow \mathbb{C}_{(n)}$ defined in an open subset $U$ of $\mathbb{R}^{n+1}$ and an open subset $\Omega$ of $U$ such that the closure $\bar{\Omega}$ of $\Omega$ is contained in $U$, and the boundary $\partial \Omega$ of $\Omega$ is a smooth oriented $n$-manifold, then the Cauchy integral formula

$$
f(x)=\int_{\partial \Omega} G_{\omega}(x) n(\omega) f(\omega) d \mu(\omega), \quad x \in \Omega
$$

is valid. Here $n(\omega)$ is the outward unit normal at $\omega \in \partial \Omega$ and $\mu$ is the volume measure of the oriented manifold $\partial \Omega$.

By analogy with formula (1), one would like to define

$$
f(A)=\int_{\partial \Omega} G_{\omega}(A) n(\omega) f(\omega) d \mu(\omega)
$$

for the pair $A=\left(A_{1}, A_{2}\right)$ of bounded linear operators on a Banach space $X$. Under the asssumption that $\sigma\left(A_{1} \xi_{1}+A_{1} \xi_{2}\right)$ is real for all $\xi_{1}, \xi_{2} \in \mathbb{R}$, we define

$$
G_{\omega}(A)=-\frac{\operatorname{sgn}\left(y_{0}\right)}{8 \pi^{2}} \int_{S_{1}}\left(\langle y I-A, s\rangle-y_{0} s I\right)^{-2} d s
$$

for $\omega=y_{0} e_{0}+y, y_{0} \in \mathbb{R} \backslash\{0\}, y \in \mathbb{R}^{2}\left[6\right.$, Lemma 2.5]. Here $S_{1}$ is the unit circle centred at zero and $d s$ is arclength measure.

The expression $\left(\langle y I-A, s\rangle-y_{0} s I\right)^{-2}, s \in S_{1}$, is understood in the sense of inversion in the module $\mathcal{L}(X) \otimes \mathbb{C}_{(2)}$, that is,

$$
\begin{aligned}
& \left(\langle y I-A, s\rangle-y_{0} s I\right)^{-2} \\
& \quad=\left(\langle y I-A, s\rangle+y_{0} s I\right)^{2}\left(\langle y I-A, s\rangle^{2}+y_{0}^{2} I\right)^{-2} \\
& \quad=\left[\left(\langle y I-A, s\rangle^{2}-y_{0}^{2} I\right)+2 s y_{0}\langle y I-A, s\rangle\right]\left(\langle y I-A, s\rangle^{2}+y_{0}^{2} I\right)^{-2} .
\end{aligned}
$$


The calculation makes sense provided that $\sigma(\langle A, \xi\rangle) \subset \mathbb{R}$ for every $\xi \in \mathbb{R}^{2}$, for then the operator $\left(\langle y I-A, s\rangle^{2}+y_{0}^{2} I\right)$ is invertible in $\mathcal{L}(X)$ by the spectral mapping theorem. To check that $G_{\omega}(A)$ is the same element of $\mathcal{L}(X) \otimes \mathbb{C}_{(2)}$ as defined by a series expansion for large $|\omega|$, it suffices to check that the function $(t, y) \longmapsto$ $G_{y+y_{0} e_{0}}(t A)$ satisfies a differential equation whose solution is given by the correct expansion at $t=1[6$, Lemma 2.5]. Then the set $\gamma(A)$ of singularities of $\omega \longmapsto$ $G_{\omega}(A)$ is a nonempty compact subset of $\{0\} \times \mathbb{R}^{2} \equiv \mathbb{R}^{2}$.

In the case that $f$ is a complex-valued analytic function of two real variables in an open neighbourhood of the compact subset $\gamma(A)$ of $\mathbb{R}^{2}$, we obtain an operator $f(A) \in \mathcal{L}(X)$ from formula (6) by extending $f$ monogenically to an open neighbourhood of $\{0\} \times \gamma(A)$ in $\mathbb{R}^{3}[6$, Theorem 3.5 (iv)].

The subset $\gamma(A)$ of $\mathbb{R}^{2}$ is the monogenic spectrum of the pair $A=\left(A_{1}, A_{2}\right)$. In the case that $A_{1}, A_{2}$ are selfadjoint, $\gamma(A)$ coincides with the support of the Weyl functional calculus for $A[5]$. As is usual in spectral theory, $\gamma(A)$ is just the set of singularities of the Cauchy kernel $\omega \longmapsto G_{\omega}(A)$ in the same way that the spectrum of a single operator is the set of singularities of its resolvent family. Some facts about the monogenic functional calculus and its application to operator theory and PDE are collected in [4].

\section{THE RIESZ-DUNFORD AND MONOGENIC FUNCTIONAL CALCULI}

Let $J: \mathbb{R}^{2} \rightarrow \mathbb{C}$ be an affine map given by $J\left(x_{1}, x_{2}\right)=a x_{1}+b x_{2}+c, x_{1}, x_{2} \in \mathbb{R}$, for some complex numbers $a, b, c$. For any pair $A=\left(A_{1}, A_{2}\right)$ of bounded linear operators acting on the Banach space $X$, abusing the notation, we write $J A$ for the linear operator $a A_{1}+b A_{2}+c I$. For any subset $B$ of $\mathbb{R}^{2}$, the image $\{J x: x \in B\}$ of $B$ by $J$ is written as $J B$.

A basic property of the Clifford algebra $\mathbb{C}_{(n)}, n=1,2, \ldots$, is that a nonzero vector $x \in \mathbb{C}^{n+1}$ is invertible in $\mathbb{C}_{(n)}$, for we may write $x^{-1}=\bar{x} /|x|^{2}$. Expressing $x \in \mathbb{C}^{n+1} \backslash\{0\}$ as $x=z_{0} e_{0}+z_{1} e_{1}+\cdots+z_{n} e_{n}$, the conjugate vector $\bar{x}$ is given by $\bar{x}=\overline{z_{0}} e_{0}-\overline{z_{1}} e_{1}-\cdots-\overline{z_{n}} e_{n}$ and $|x|^{2}=\left|z_{0}\right|^{2}+\left|z_{1}\right|^{2}+\cdots+\left|z_{n}\right|^{2}=x \bar{x}$ is the square of the Euclidean norm of $x$.

Theorem 1. Let $A_{1}, A_{2}$ be bounded linear operators acting on the complex Banach space $X$ with the property that $\sigma\left(A_{1} \xi_{1}+A_{2} \xi_{2}\right) \subset \mathbb{R}$ for all $\xi \in \mathbb{R}^{2}$. Let $\gamma(A)$ be the monogenic spectrum of the pair $A=\left(A_{1}, A_{2}\right)$ and let $J: \mathbb{R}^{2} \rightarrow \mathbb{C}$ be an affine map.

For every $\lambda=\lambda_{0} e_{0}+\lambda_{1} e_{1}+\lambda_{2} e_{2} \in \mathbb{C}^{3}$, let

$$
\varphi_{\lambda}(x)=\left(\lambda-(J x) e_{0}\right)^{-1} \in \mathbb{C}_{(2)}
$$

for all $x=\left(x_{1}, x_{2}\right) \in \mathbb{R}^{2}$ such that $(J x) e_{0} \neq \lambda$.

Then $\lambda I-(J A) e_{0}$ is invertible in $\mathcal{L}(X) \otimes \mathbb{C}_{(2)}$ for every $\lambda \in \mathbb{C}^{3} \backslash J \gamma(A)$. Moreover, if $\varphi_{\lambda}(A)$ is defined by formula (6) for $\lambda \in \mathbb{C}^{3} \backslash J \gamma(A)$, then the equality

$$
\varphi_{\lambda}(A)=\left(\lambda I-(J A) e_{0}\right)^{-1}, \quad \lambda \in \mathbb{C}^{3} \backslash J \gamma(A),
$$


is valid. In particular, $\sigma(J A) \subset J \gamma(A)$.

Proof. For each $j=1,2$, the unique left and right monogenic extension of the coordinate function $x \longmapsto x_{j}, x \in \mathbb{R}^{2}$, is $\omega \longmapsto \omega_{j} e_{0}-\omega_{0} e_{j}, \omega=\left(\omega_{0}, \omega_{1}, \omega_{2}\right) \in \mathbb{R}^{3}$.

Suppose that $a, b, c$ are complex numbers for which $J\left(x_{1}, x_{2}\right)=a x_{1}+b x_{2}+c$ for all $x_{1}, x_{2} \in \mathbb{R}$. For every $\lambda=\lambda_{0} e_{0}+\lambda_{1} e_{1}+\lambda_{2} e_{2} \in \mathbb{C}^{3}$, let $\tilde{\varphi}_{\lambda}$ be the $\mathbb{C}_{(2)}$-valued function given by

$$
\begin{aligned}
\tilde{\varphi}_{\lambda}(\omega) & =\frac{\left(\lambda-a\left(\omega_{1} e_{0}-\omega_{0} e_{1}\right)-b\left(\omega_{2} e_{0}-\omega_{0} e_{2}\right)-c e_{0}\right)^{-1}}{\lambda_{0}-\left(a \omega_{1}+b \omega_{2}+c\right) e_{0}-\overline{\lambda_{1}-a \omega_{0}} e_{1}-\overline{\lambda_{2}-b \omega_{0}} e_{2}} \\
& =\frac{\lambda_{0}-\left.\left(a \omega_{1}+b \omega_{2}+c\right)\right|^{2}+\left|\lambda_{1}-a \omega_{0}\right|^{2}+\left|\lambda_{2}-b \omega_{0}\right|^{2}}{\mid \lambda^{2}}
\end{aligned}
$$

for all $\omega=\left(\omega_{0}, \omega_{1}, \omega_{2}\right) \in \mathbb{R}^{3}$ for which the denominator is nonzero.

The restriction of $\tilde{\varphi}_{\lambda}$ to $\mathbb{R}^{2}$ is equal to $\varphi_{\lambda}$, that is, on putting $\omega_{0}=0$. For each $\lambda \in \mathbb{C}^{3} \backslash J \gamma(A)$, the function $\tilde{\varphi}_{\lambda}$ takes its values in the linear subspace spanned by $e_{0}, e_{1}, e_{2}$ in $\mathbb{C}_{(2)}$ and is left and right monogenic in an open neighbourhood

$U_{\lambda}=\left\{\left(\omega_{0}, \omega_{1}, \omega_{2}\right) \in \mathbb{R}^{3}:\left|\lambda_{0}-\left(a \omega_{1}+b \omega_{2}+c\right)\right|^{2}+\left|\lambda_{1}-a \omega_{0}\right|^{2}+\left|\lambda_{2}-b \omega_{0}\right|^{2}>0\right\}$

of $\{0\} \times \gamma(A)$ in $\mathbb{R}^{3}$.

Let $\Omega$ be any open subset of $U_{\lambda}$ in $\mathbb{R}^{3}$ containing $\{0\} \times \gamma(A)$ such that the closure $\bar{\Omega}$ of $\Omega$ is contained in $U_{\lambda}$, and the boundary $\partial \Omega$ of $\Omega$ is a smooth oriented $n$-manifold. Then the operator $\varphi_{\lambda}(A):=\tilde{\varphi}_{\lambda}(A)$ is defined by the Cauchy integral formula (6).

The Neumann series expansion

$$
\lambda^{-1} I+\sum_{k=1}^{\infty} \lambda^{-(k+1)}(J A)^{k}
$$

of $\left(\lambda I-(J A) e_{0}\right)^{-1}$ converges in $\mathcal{L}(X) \otimes \mathbb{C}_{(2)}$ for $\lambda \in \mathbb{C}^{3}$ with $|\lambda|$ large enough. Moreover, the sums

$$
\begin{aligned}
& \varphi_{\lambda}(x)=\sum_{k=0}^{\infty} \lambda^{-(k+1)}(J x)^{k} \\
& \tilde{\varphi}_{\lambda}(\omega)=\sum_{k=0}^{\infty} \lambda^{-(k+1)}\left(a\left(\omega_{1} e_{0}-\omega_{0} e_{1}\right)+b\left(\omega_{2} e_{0}-\omega_{0} e_{2}\right)+c e_{0}\right)^{k}
\end{aligned}
$$

converge uniformly in $\mathbb{C}_{(2)}$ as $x \in \mathbb{R}^{2}$ and $\omega \in \mathbb{R}^{3}$ range over compact sets and $\lambda$ is outside a sufficiently large ball in $\mathbb{C}^{3}$. For each $k=0,1, \ldots$, set

$$
\begin{aligned}
& p_{k}(x)=(J x)^{k}, \quad x \in \mathbb{R}^{2}, \\
& \tilde{p}_{k}(\omega)=\left(a\left(\omega_{1} e_{0}-\omega_{0} e_{1}\right)+b\left(\omega_{2} e_{0}-\omega_{0} e_{2}\right)+c e_{0}\right)^{k}, \quad \omega \in \mathbb{R}^{3} .
\end{aligned}
$$

Then $\tilde{p}_{k}$ is the unique left and right monogenic extension of the polynomial $p_{k}$ to all of $\mathbb{R}^{3}$, for each $k=0,1, \ldots$. 
Now $\varphi_{\lambda}(A)=\tilde{\varphi}_{\lambda}(A)$, by definition, for all $\lambda \in \mathbb{C}^{3} \backslash J \gamma(A)$. According to [6, Theorem 3.5 (ii)], the equality

$$
p_{k}(A)=\tilde{p}_{k}(A)=(J A)^{k}
$$

holds for all $k=0,1,2 \ldots$, so by the continuity of the monogenic functional calculus $f \longmapsto f(A)[6$, Proposition 3.3], we have

$$
\varphi_{\lambda}(A)=\lambda^{-1} I+\sum_{k=1}^{\infty} \lambda^{-(k+1)}(J A)^{k}=\left(\lambda I-(J A) e_{0}\right)^{-1},
$$

that is,

$$
\left(\lambda I-(J A) e_{0}\right) \varphi_{\lambda}(A)=\varphi_{\lambda}(A)\left(\lambda I-(J A) e_{0}\right)=I,
$$

for all $\lambda \in \mathbb{C}^{3}$ with $|\lambda|$ sufficiently large.

The linear operator $\varphi_{\lambda}(A)$ is defined by formula (6) for all $\lambda \in \mathbb{C}^{3} \backslash J \gamma(A)$ and by differentiation under the integral (6), we see that $\lambda \longmapsto \varphi_{\lambda}(A), \lambda \in \mathbb{C}^{3} \backslash$ $J \gamma(A)$, is a holomorphic $\mathcal{L}(X) \otimes \mathbb{C}_{(2)}$-valued function. Because $\mathbb{C}^{3} \backslash J \gamma(A)$ is a connected set, it follows that equation (9) holds for all $\lambda \in \mathbb{C}^{3} \backslash J \gamma(A)$, so that $\varphi_{\lambda}(A)=\left(\lambda I-(J A) e_{0}\right)^{-1}$. In particular, equation (9) holds for all $\lambda=\lambda_{0} e_{0}$ with $\lambda_{0} \in \mathbb{C} \backslash J \gamma(A)$ and the resolvent set of the bounded linear operator $J A$ contains $\mathbb{C} \backslash J \gamma(A)$.

Applying Theorem 1 to the usual identification of the plane $\mathbb{R}^{2}$ with $\mathbb{C}$, we have the following improvement of [1, Theorem 5.1].

Corollary. Let $A_{1}, A_{2}$ be bounded linear operators acting on the Banach space $X$ with the property that $\sigma\left(A_{1} \xi_{1}+A_{2} \xi_{2}\right) \subset \mathbb{R}$ for all $\xi \in \mathbb{R}^{2}$. Let $\gamma(A)$ be the monogenic spectrum of the pair $A=\left(A_{1}, A_{2}\right)$ and let $j: \mathbb{R}^{2} \rightarrow \mathbb{C}$ be defined by $j\left(x_{1}, x_{2}\right)=x_{1}+i x_{2}$ for $x_{1}, x_{2} \in \mathbb{R}^{2}$. Then $\sigma\left(A_{1}+i A_{2}\right) \subset j \gamma(A)$. Furthermore, if $f$ is a holomorphic function defined in a neighbourhood $U$ of $j \gamma(A)$ in $\mathbb{C}$ and $(f \circ j)(A)$ is defined by formula (6), then

$$
(f \circ j)(A)=\frac{1}{2 \pi i} \int_{C}\left(\lambda I-\left(A_{1}+i A_{2}\right)\right)^{-1} f(\lambda) d \lambda
$$

for any simple closed contour $C$ about $\sigma\left(A_{1}+i A_{2}\right)$ contained in $U$.

Proof. The identification $j$ of $\mathbb{R}^{2}$ with $\mathbb{C}$ is an affine map, so the inclusion $\sigma\left(A_{1}+\right.$ $\left.i A_{2}\right) \subset j \gamma(A)$ is a special case of Theorem 1. The equality (10) would follow from Runge's Theorem, as in the proof of [1, Theorem 5.1], if $f$ were holomorphic on a simply connected domain containing $j \gamma(A)$, but even for two $(3 \times 3)$ matrices, it may happen that $f$ is holomorphic on an open neighbourhood of $j \gamma(A)$ that is not simply connected in $\mathbb{C}$, see $[7$, Figure 3]. The key is to approximate $f \circ j$ by monogenic polynomials in one higher dimension. 
Let $H_{M}(\gamma(A))$ denote the space of all $\mathbb{C}$-valued functions which are real analytic in a neighbourhood of the compact subset $\gamma(A)$ of $\mathbb{R}^{2}$. We give $H_{M}(\gamma(A))$ the topology inherited from the space $M\left(\gamma(A), \mathbb{C}_{(n)}\right)$ of left monogenic functions defined in a neighbourhood of $\gamma(A)$ [4, Section 3.8] in which $H_{M}(\gamma(A))$ is embedded by the Cauchy-Kowalewski extension mapping [4, Section 3.5]. A sequence of functions $f_{n}, n=1,2, \ldots$, converges in $H_{M}(\gamma(A))$ if there exists an open neighbourhood $V$ of $\gamma(A)$ in $\mathbb{R}^{n+1}$ such that the left monogenic extension $\tilde{f}_{n}$ of $f_{n}$ to $V$ exists for each $n=1,2, \ldots$ and converges uniformly on compact subsets of $V$ in $\mathbb{R}^{n+1}$ as $n \rightarrow \infty$.

Let $\Gamma$ be a closed contour in $U$ such that $\Gamma$ winds once about each point $z \in j \gamma(A)$ but not around any point not in $U$. The function $\lambda \longmapsto(\lambda-j(\cdot))^{-1} f(\lambda)$, $\lambda \in \Gamma$, is continuous from $\Gamma$ into $H_{M}(\gamma(A))$ because according to equation (8),

$$
\lambda e_{0}-\left(x_{1} e_{0}-x_{0} e_{1}+i\left(x_{2} e_{0}-x_{0} e_{2}\right)\right)
$$

is invertible in $\mathbb{C}_{(2)}$ for all $\lambda \in \Gamma$ and all $x=\left(x_{0}, x_{1}, x_{2}\right)$ belonging to an open set in $\mathbb{R}^{3}$ containing $\{0\} \times \gamma(A)$. Let $T: \phi \longmapsto \phi(A), \phi \in H_{M}(\gamma(A))$, be the mapping defined by formula (6). According to [4, Proposition 4.19, Theorem 4.22], the linear mapping $T$ is continuous from $H_{M}(\gamma(A))$ into $\mathcal{L}(X)$. An appeal to the complex Cauchy integral formula and properties of the Bochner integral (see [5] for a similar argument) give

$$
\begin{aligned}
(f \circ j)(A) & =T(f \circ j) \\
& =\frac{1}{2 \pi i} T \int_{\Gamma}(\lambda-j(\cdot))^{-1} f(\lambda) d \lambda \\
& =\frac{1}{2 \pi i} \int_{\Gamma} T\left((\lambda-j(\cdot))^{-1}\right) f(\lambda) d \lambda \\
& =\frac{1}{2 \pi i} \int_{\Gamma} T\left(\varphi_{\lambda}\right) f(\lambda) d \lambda \\
& =\frac{1}{2 \pi i} \int_{\Gamma}\left(\lambda I-\left(A_{1}+i A_{2}\right)\right)^{-1} f(\lambda) d \lambda,
\end{aligned}
$$

where the last equality follows from Theorem 1 . The desired representation (10) now follows, because $\Gamma$ and $C$ are homologous in $U \backslash \sigma\left(A_{1}+i A_{2}\right)$.

Example 1. For the two Pauli matrices $A_{1}=\left(\begin{array}{cc}1 & 0 \\ 0 & -1\end{array}\right)$ and $A_{2}=\left(\begin{array}{ll}0 & 1 \\ 1 & 0\end{array}\right)$, we have $\sigma\left(A_{1}+i A_{2}\right)=\{0\}$ and $\gamma(A)=\left\{x \in \mathbb{R}^{2}:|x| \leq 1\right\}$ [4, Example 4.1].

Example 2. The pair $A=\left(A_{1}, A_{2}\right)$ of $3 \times 3$ matrices

$$
A_{1}=\left(\begin{array}{ccc}
1 & 0 & 0 \\
0 & -1 & 0 \\
0 & 0 & -\frac{3}{2}
\end{array}\right), \quad A_{2}=\left(\begin{array}{ccc}
0 & 0 & 0 \\
0 & 0 & 1 \\
0 & 1 & 0
\end{array}\right)
$$


has a joint eigenvalue $(1,0)$ and the reduced matrices

$$
A_{1}^{\prime}=\left(\begin{array}{cc}
-1 & 0 \\
0 & -\frac{3}{2}
\end{array}\right), \quad A_{2}^{\prime}=\left(\begin{array}{ll}
0 & 1 \\
1 & 0
\end{array}\right)
$$

may be expressed in terms of the identity matrix $I$ and the Pauli matrices

$$
\sigma_{1}=\left(\begin{array}{cc}
0 & 1 \\
1 & 0
\end{array}\right), \quad \sigma_{3}=\left(\begin{array}{cc}
1 & 0 \\
0 & -1
\end{array}\right)
$$

as $\left(A_{1}^{\prime}, A_{2}^{\prime}\right)=\left(\frac{1}{4} \sigma_{3}-\frac{5}{4} I, \sigma_{1}\right)$. Let $J: \mathbb{R}^{2} \rightarrow \mathbb{R}^{2}$ denote the affine map $J\left(x_{1}, x_{2}\right)=$ $\left(\frac{1}{4} x_{1}-\frac{5}{4}, x_{2}\right), x_{1}, x_{2} \in \mathbb{R}$ and let $D$ denote the closed unit disk centred at zero in $\mathbb{R}^{2}$. Then $\gamma\left(\sigma_{3}, \sigma_{1}\right)=D[4$, Example 4.10]. By the invariance properties of the Weyl calculus [1], $\gamma(A)$ is the union of the elliptical region $J D$ centred at $\left(-\frac{5}{4}, 0\right)$ and the isolated point $\{(1,0)\}$. Because $\sigma\left(A_{1}+i A_{2}\right)=\left\{1,-\frac{5}{4} \pm \frac{\sqrt{15}}{4} i\right\}$ and $\left(-\frac{5}{4}, \pm \frac{\sqrt{15}}{4}\right) \in J D$, the spectrum $\sigma\left(A_{1}+i A_{2}\right)$ of $A_{1}+i A_{2}$ is contained in the image $j \gamma(A)$ of $\gamma(A)$ in $\mathbb{C}$, see [7, Figure 2].

Example 3. The pair $A=\left(A_{1}, A_{2}\right)$ of $3 \times 3$ matrices

$$
A_{1}=\left(\begin{array}{ccc}
1 & 0 & 0 \\
0 & -1 & 0 \\
0 & 0 & -\frac{3}{2}
\end{array}\right), \quad A_{2}=\left(\begin{array}{ccc}
0 & 0 & 1 \\
0 & 0 & 1 \\
1 & 1 & 0
\end{array}\right)
$$

has no proper joint invariant subspaces, that is, there are no joint eigenvectors. The image $j \gamma(A)$ of $\gamma(A)$ in $\mathbb{C}$ consists of the numerical range of the matrix $A_{1}+i A_{2}$ less an interior region bounded by straight lines $j L_{s}$ satisfying $\langle x, s\rangle \in \sigma\left(A_{1} s_{1}+A_{2} s_{2}\right)$ for $x \in L_{s}$ and $s \in \mathbb{R}^{2}$ with $|s|=1$, see [7, Figure 3], from which it is clear that the eigenvalues $\sigma\left(A_{1}+i A_{2}\right) \approx\{0.6359991610,-1.067999581 \pm 1.103571734 i\}$ of $A_{1}+i A_{2}$ are contained in $j \gamma(A)$, as required by the Corollary to Theorem 1 .

\section{REFERENCES}

1. R.F.V. Anderson, "The Weyl functional calculus", J. Funct. Anal. 4 (1969), 240-267.

2. B. Jefferies, "The Weyl calculus for hermitian matrices", Proc. Amer. Math. Soc. 124 (1996), 121-128.

3. B. JefFeries, "Exponential bounds for noncommuting systems of matrices", Studia Math. 144 (2001), 197-207.

4. B. Jefferies, "Spectral properties of noncommuting operators", Lecture Notes in Mathematics 1843, Springer-Verlag, 2004.

5. B. Jefferies And A. McIntosh, "The Weyl calculus and Clifford analysis", Bull. Austral. Math. Soc. 57 (1998), 329-341.

6. B. Jefferies, A. Mcintosh and J. Picton-Warlow, "The monogenic functional calculus", Studia Math. 136 (1999), 99-119. 
7. B. Jefferies and B. Straub, "Lacunas in the support of the Weyl functional calculus for pairs of hermitian matrices", J. Austral. Math. Soc. 75 (2003), 85-124.

8. R.M. Range, Holomorphic Functions and Integral Representations in Several Complex Variables, Graduate Texts in Mathematics 108, Springer-Verlag, New York, 1986.

9. M.E. TAYLOR, "Functions of several self-adjoint operators", Proc. Amer. Math. Soc. 19 (1968), 91-98.

B. Jefferies: School of Mathematics, The University of New South Wales, Sydney, NSW 2052, Australia

E-mail: b.jefferies@unsw.edu.au 